APS

physics

This is the accepted manuscript made available via CHORUS. The article has been published as:

\title{
Recombination-Enhanced Surface Expansion of Clusters in Intense Soft X-Ray Laser Pulses
}

Daniela Rupp, Leonie Flückiger, Marcus Adolph, Tais Gorkhover, Maria Krikunova, Jan Philippe Müller, Maria Müller, Tim Oelze, Yevheniy Ovcharenko, Benjamin Röben, Mario Sauppe, Sebastian Schorb, David Wolter, Rolf Mitzner, Michael Wöstmann, Sebastian Roling, Marion Harmand, Rolf Treusch, Mathias Arbeiter, Thomas Fennel, Christoph

Bostedt, and Thomas Möller

Phys. Rev. Lett. 117, 153401 - Published 7 October 2016

DOI: 10.1103/PhysRevLett.117.153401 


\title{
Observation of recombination-enhanced surface expansion of clusters in intense soft $\mathrm{x}$-ray laser pulses
}

\author{
Daniela Rupp, ${ }^{1, *}$ Leonie Flückiger,,${ }^{1,2}$ Marcus Adolph, ${ }^{1}$ Tais Gorkhover, ${ }^{1,3}$ Maria Krikunova, ${ }^{1}$ Jan \\ Philippe Müller, ${ }^{1}$ Maria Müller,${ }^{1}$ Tim Oelze, ${ }^{1}$ Yevheniy Ovcharenko, ${ }^{1}$ Benjamin Röben, ${ }^{1}$ Mario Sauppe,${ }^{1}$ \\ Sebastian Schorb, ${ }^{3,1}$ David Wolter, ${ }^{1}$ Rolf Mitzner, ${ }^{4}$ Michael Wöstmann, ${ }^{5}$ Sebastian Roling, ${ }^{5}$ Marion \\ Harmand, ${ }^{6}$ Rolf Treusch, ${ }^{6}$ Mathias Arbeiter, ${ }^{7}$ Thomas Fennel, ${ }^{7}{ }^{\dagger}$ Christoph Bostedt, ${ }^{3,8}$ and Thomas Möller ${ }^{1}$ \\ ${ }^{1}$ IOAP, Technische Universität Berlin, Hardenbergstraße 36, 10623 Berlin, Germany \\ ${ }^{2}$ ARC CoE AMI, La Trobe University, Bundoora, Victoria 3086, Australia \\ ${ }^{3} L C L S$, SLAC, 2575 Sand Hill Road, Menlo Park, CA 94025, USA \\ ${ }^{4}$ Helmholtz-Zentrum Berlin, Hahn-Meitner-Platz 1, 14109 Berlin, Germany \\ ${ }^{5}$ Universität Münster, Wilhelm-Klemm-Straße 10, 48149 Münster, Germany \\ ${ }^{6}$ FLASH, DESY, Notkestraße 85, 22605 Hamburg, Germany \\ ${ }^{7}$ Universität Rostock, Albert-Einstein-Str. 23, 18059 Rostock, Germany \\ ${ }^{8}$ Argonne National Laboratory, 9700 S. Cass Avenue, Argonne, IL 60439, USA
}

(Dated: September 30, 2016)

\begin{abstract}
We studied the nanoplasma formation and explosion dynamics of single large xenon clusters in ultrashort, intense x-ray free electron laser pulses via ion spectroscopy. The simultaneous measurement of single-shot diffraction images enabled a single-cluster analysis that is free from any averaging over the cluster size and laser intensity distributions. The measured charge state-resolved ion energy spectra show narrow distributions with peak positions that scale linearly with final ion charge state. These two distinct signatures are attributed to highly efficient recombination that eventually leads to the dominant formation of neutral atoms in the cluster. The measured mean ion energies exceed the value expected without recombination by more than an order of magnitude, indicating that the energy release resulting from electron-ion recombination constitutes a previously unnoticed nanoplasma heating process. This conclusion is supported by results from semiclassical molecular dynamics simulations.
\end{abstract}

The advent of ultrashort and extremely bright x-ray pulses from free electron lasers (FEL) [1-3] has opened up unprecedented routes to study the structure and dynamics of matter, ranging from structural analysis of nanosamples [4-8] and viruses [9, 10], over molecular reaction dynamics [11] to fundamental properties of matter under extreme conditions [12]. Because of their scalable size and simple structure, atomic clusters in the gas phase have proven to be ideal model systems for studying extreme laser-matter interaction across all wavelength regimes [13-20]. Starting with the very first experiment at an FEL in the short-wavelength range [21], studies on atomic clusters have contributed significantly to a fundamental understanding of ultrafast $\mathrm{x}$-ray induced dynamics in finite systems [22-40].

The possibility to image single particles via single-shot $\mathrm{x}$-ray diffraction enables novel insight into the formation and morphology of clusters [7, 41-43] as well as ultrafast transient changes of their electronic properties [44]. Further, since both initial cluster size and laser intensity (or fluence) are encoded in the diffraction image [44, 45], the combination of $\mathrm{x}$-ray imaging and ion spectroscopy enables studying intense $\mathrm{x}$-ray laser-cluster interactions with unprecedented control over these experimental parameters. The resulting possibility to circumvent the usually unavoidable averaging over the focal intensity profile and the cluster size distribution [46-48] provides striking new insights, as demonstrated recently by the observation of the exclusive emission of highly charged ions from xenon clusters under hard x-ray pulses [45]. In the current work, we utilize this combined approach to study the charge state selective ion expansion of single large xenon clusters $(R=180-600 \mathrm{~nm})$ induced by intense femtosecond soft x-ray pulses $(h \nu=91 \mathrm{eV}$, $I \leq 5 \times 10^{14} \mathrm{~W} / \mathrm{cm}^{2}$ ). The systematic analysis of the intensity and cluster size dependent explosion dynamics via the charge state-resolved ion kinetic energy distributions yields detailed information about the electron-ion relaxation dynamics in the expanding nanoplasma. Most importantly, we identify the energy transfer resulting from electron-ion recombination, i.e., the energy release to the nanoplasma due to the electron capture process, as an important and previously unnoticed nanoplasma heating process.

For the large clusters considered here and the high ionization cross section of xenon [49] at $91 \mathrm{eV}$, the cluster expansion can be expected to proceed deeply in the hydrodynamic regime: Photoelectrons become trapped rapidly in the emerging cluster Coulomb field [50], forming a dense and virtually neutral nanoplasma. As a result the nanoplasma expands via conversion of thermal electron energy to radial ionic motion [29], while contributions from Coulomb explosion due to the net cluster charge are negligible when compared to the thermal contribution. The mechanism is based on the thermal spill-out of the electron cloud over the 


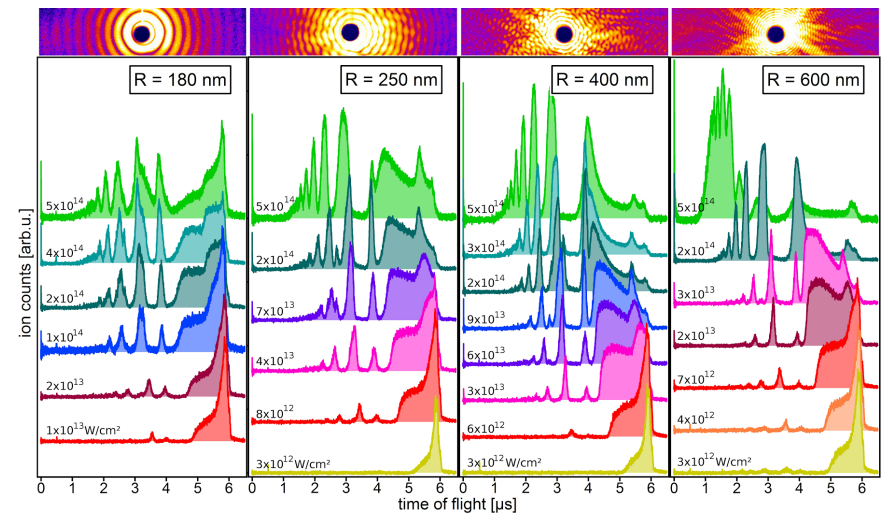

FIG. 1. Ion time-of-flight spectra of single large clusters (radii of $180( \pm 30) \mathrm{nm}, 250( \pm 40) \mathrm{nm}, 400( \pm 50) \mathrm{nm}$, and $600( \pm 50) \mathrm{nm})$ sorted by cluster size and laser intensity, derived by comparison with Mie simulations, see text. The spectra are sorted from bottom to top by increasing image brightness, corresponding colors reflect similar estimated FEL intensities. Offsets are applied for better visibility. Please note that the $400 \mathrm{~nm}$-ion spectra constitute a representative selection out of 94 spectra, while in the other size regimes only 5-10 events were collected. Given FEL intensity values at the positions of the clusters have to be considered as orientation only, as the intensity assignment may be compromised by detector nonlinearities [42] and intensity dependent ultrafast electronic changes in the cluster [44].

cluster boundary due to the non-zero temperature of the quasi-free electrons, resulting in a partially unscreened ionic surface that is expelled from the cluster. The cluster expansion in the hydrodynamic regime typically leads to energy spectra that are continuous and peaked near zero kinetic energy [29]. As collisional heating (inverse bremsstrahlung) is negligible for the considered laser wavelength, the electron temperature can be approximated from the excess energy $E_{\text {excess }}$ of the photoelectrons and secondary Auger electrons [23]. Assuming a complete conversion of the thermal electron energy to ionic motion in the hydrodynamic expansion and approximating the electrons as an ideal gas, an upper bound for the average ion kinetic energy is given by $\bar{E}=\frac{3}{2} k_{B} T_{e} \bar{Q}[47]$ where $\bar{Q}$ is the (dimensionless) average charge of the atomic ions, also corresponding to the number of quasi-free electrons per atom in a quasi-neutral nanoplasma.

In our experiment we observe strong deviations from this expected behavior: (i) The charge state-resolved energy distributions are sharp and peaked at non-zero energies for all ion charge states, i.e. slow ions are effectively absent. (ii) The average energy per average charge state exceeds the above estimate by a factor of up to 25 . As discussed in detail below, these findings support that the efficient electron-ion recombination during expansion is accompanied by the release of energy from recombining electrons to the nanoplasma and, eventually, to only a subset of the ions.

Soft x-ray pulses from FLASH (Free-electron $L A S$ er in $H$ amburg) with $\approx 100$ fs pulse duration were focused to peak intensities of up to $5 \times 10^{14} \mathrm{~W} / \mathrm{cm}^{2}$ and overlapped with a dilute beam of large xenon clusters from a nozzle expansion. The elastically scattered photons from single clusters were measured shot-to-shot by a large area detector. An ion time-of-flight (TOF) spectrometer with a small entrance aperture was positioned perpendicular to FEL and cluster beam axes. Further details of the experimental setup are described elsewhere [41].

From the single-cluster images, the cluster size was determined by comparison with Mie simulations (cf. [41]). A selection of resulting size-dependent single-cluster ion spectra is presented in Fig. 1. The respective FEL intensities are estimated from the scattering images using Mie simulations and assuming that most intense hits correspond to the focal peak intensity. Note that the intensity assignment may be compromised by detector nonlinearities [42] and intensity dependent ultrafast electronic changes in the cluster [44].

The conversion of the ion TOF spectra to energy distributions proceeds as demonstrated in Fig. 2a) and b) on the example of the most intense $R=400 \mathrm{~nm}$ ion spectrum. The intense peak at $t=0$ in Fig. 2a) results from scattered light, followed by features from atomic ions in different charge states. Compared to atomic xenon gas, the ion peaks from clusters are shifted to shorter flight times because of their kinetic energy. The TOF spectra are converted to kinetic energy spectra using a simulation-based conversion function that accounts for the spectrometer transmission, see supplemental materials [51] for details. Note that 'back-peaks' (typical features of this spectrometer type, formed by ions starting in the direction opposite to the drift-tube aperture and becoming reversed in the acceleration field, see [52] and supplemental section [51]) have been identified by the simulations and excluded in the further analysis.

The corresponding $\mathrm{Xe}^{q+}$ kinetic energy spectra up to $q=5$ reveal three main features (Fig. $2 \mathrm{~b}$ ). (i) If rescaled by their charge state, the distributions for all charge states are peaked for similar $E_{k i n} / q$ values and show a similar cutoff behavior at the high energy side. (ii) The contribution from $\mathrm{Xe}^{1+}$ is dominant. (iii) Only the $\mathrm{Xe}^{1+}$ spectra show a low energy tail that extends to zero energy. These signatures have been found to be generic for the investigated size and intensity range. We would like to stress, that the extraction of meaningful charge state resolved energy spectra is only possible for single cluster ion spectra, as the signal from many clusters of different sizes irradiated with different laser intensities would wash out the clear cut-offs of the peaks towards longer flight times (see also supplemental material [51] and [45]).

Energy spectra with isolated peaks have been predicted 

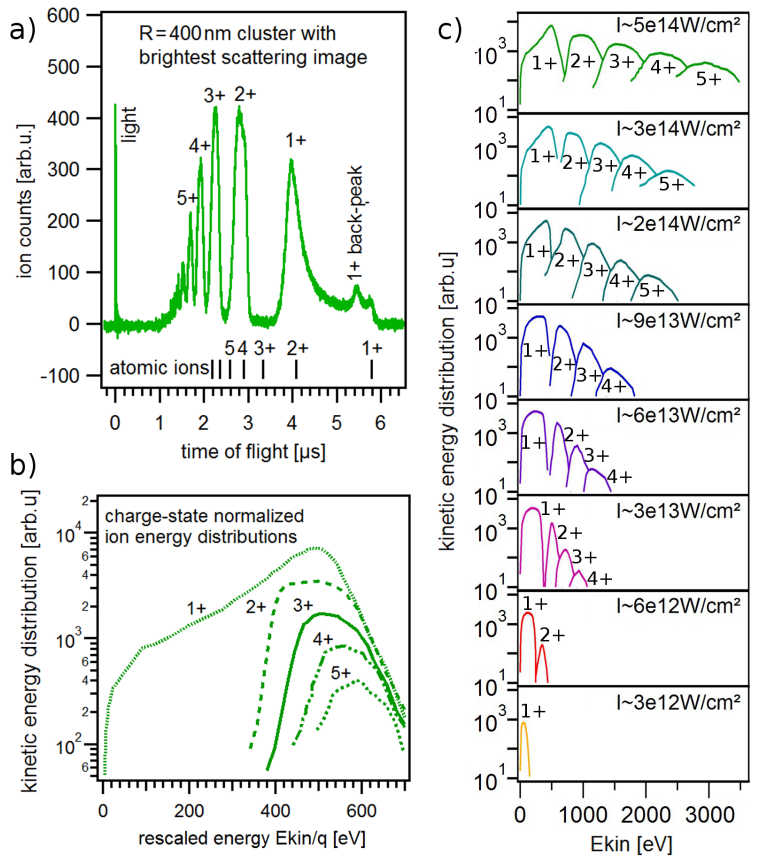

FIG. 2. a) Most intense time-of-flight spectrum of $R=400 \mathrm{~nm}$ clusters. The light peak and ion charge states up to $q=5$ are indicated. Flight-times of atomic ions are given for comparison. The $\mathrm{Xe}^{1+}$ - 'back-peak' can be observed at $5.5 \mu \mathrm{s}$ ('backpeaks' have been excluded for analysis, see supplements [51]). b) Kinetic energy distribution of each charge state for the same ion spectrum as a function of $E_{k i n}$ per charge state, corrected for the spectrometer transmission. c) Evolution of the charge state-resolved kinetic energies for $R=400 \mathrm{~nm}$ clusters irradiated with different FEL intensities. Note that the spectra show narrow energy distributions and essentially no slow ions for $q>1$. Any signal of slow ions would have been enhanced by the spectrometer transmission.

in simulations including recombination [29]. The absence of slow ions from charge states $>1+$ in our data is a signature of the predicted dynamic population transfer from high to low charge states and finally to neutral atoms in the nanoplasma expansion process $[26,29]$. The common cut-off for all charge states at the high-energy side $(\approx 700 \mathrm{eV})$ reflects an effective maximal acceleration in the nanoplasma expansion, being the second indication for efficient recombination as analyzed in detail below.

In Fig. 2c), the evolution of the charge state-resolved spectra for $R=400 \mathrm{~nm}$ with intensity reveals the gradual increase of the maximal charge states, an increasing $E_{k i n}$ per charge state ratio of the individual peaks, and the otherwise generic structure of the spectra. Similar evolutions are found for all other investigated cluster sizes. Ions with low kinetic energy are missing for $q>1$ in all cases in Fig. 2c). We would like to emphasize that the absence of slow ions is not an artifact of the spectrometer transmission, which is maximal for slow ions and has been taken into account for conversion of

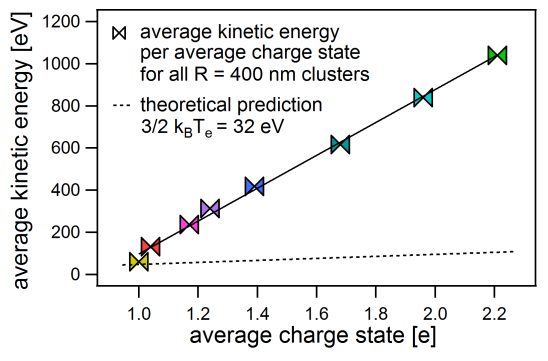

FIG. 3. Average kinetic energy $\bar{E}$ per average charge state $\bar{Q}$ measured for $R=400 \mathrm{~nm}$ clusters. Each point has been calculated from one spectrum in figure 2, as indicated by the color coding. Energy conservation demands that an upper limit for the average ion energy for pure hydrodynamic expansion, neglecting recombination, is given by $\bar{E}=(3 / 2) k_{B} T_{e} \bar{Q}[47]$. The dashed line represents the expected curve for an average electron energy of $32 \mathrm{eV}$. The slope of the measured curve corresponds to an effective electron temperature of $700 \mathrm{eV}$.

the spectra.

Finally, the charge state-resolved single-shot singlecluster energy spectra enable a quantitative analysis of the energy balance of the expansion process. Therefore, the average ion kinetic energy $\bar{E}$ for $R=400 \mathrm{~nm}$ clusters is analyzed as function of the average charge state $\bar{Q}$ of the emitted ions, see Fig. 3. Each point in Fig. 3 is calculated by averaging over the corresponding spectra in Fig. 2c), as indicated by the color coding (for details see supplemental material [51]). Fig. 3 shows a linear increase of the average ion kinetic energy with average charge with a slope of more than $700 \mathrm{eV}$ per charge state. While we expect a linear evolution $\bar{E}(\bar{Q})=\overline{E_{\text {kin }, e l}} \cdot \bar{Q} \approx E_{\text {excess }} \cdot \bar{Q}$ for a hydrodynamic expansion without recombination [47] (note that $\bar{Q}$ is the dimensionless average charge state, corresponding to the average number of electrons per atom in a quasi-neutral plasma), the steep slope would imply an unreasonably high plasma temperature of several hundred $\mathrm{eV}$, which is unphysical for the considered photon energy of $91 \mathrm{eV}$. For comparison, the predicted curve for hydroexpansion of a non-recombining plasma with an electron energy of $32 \mathrm{eV}$ (corresponding to the energy of Xe Auger electrons from absorbing $91 \mathrm{eV}$ photons [22]) is presented as a dotted line in Fig. 3.

To explain the experimental data we propose the following picture: First, direct x-ray photoionization and secondary Auger emission create high atomic charge states [31, 53]. However, a nearly neutral nanoplasma is formed because of the efficient trapping of electrons for large clusters $[29,50]$. The plasma electrons thermalize [23] and loose further energy via inelastic collisions with ions [53]. The resulting electron temperature $\left(k_{B} T_{e}<E_{\text {excess }}<\hbar \omega\right)$ causes the hydroexpansion that begins with surface ions. Only the fast fraction of the outermost ions expand sufficiently fast to avoid electron 

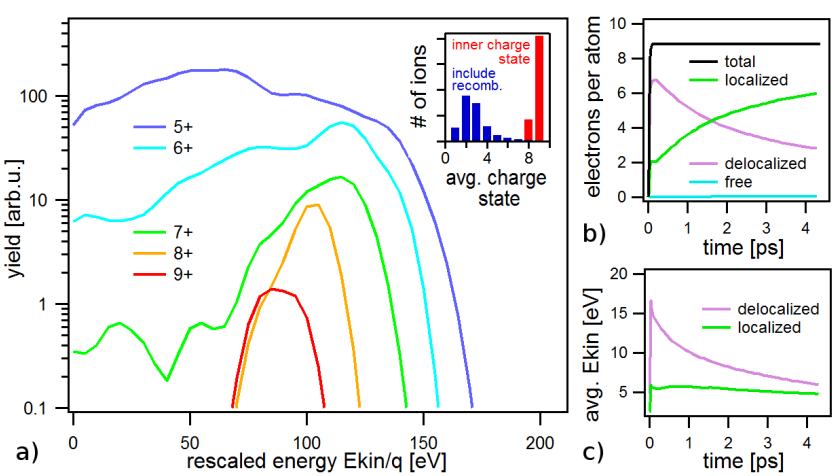

FIG. 4. Semiclassical MD simulation of $\mathrm{Xe}_{24739}$ under a $12 \mathrm{fs}$ XUV laser pulse $\left(h \nu=90 \mathrm{eV}, I=5 \times 10^{14} \mathrm{~W} / \mathrm{cm}^{2}\right)$. a) Ion spectra including recombination (see inset) after 4 ps propagation. Spectra for $q<5$ are omitted as recombination in later stages will still change their distributions substantially. Note that the spectra reflect the energy per charge state. (b,c) Time-dependent evolution of electron numbers and thermal energy of free, delocalized and localized (recombined) electrons.

capture. The slower fraction experiences a high electron density for a longer time and recombines sequentially via three-body recombination, explaining the isolated peaks in the energy spectra. The fact that also the distributions of singly charged ions show a peak at energies far beyond any physically reasonable electron temperature and a clear drop towards zero kinetic energy implies that the dominant fraction of generated ions recombines to fully neutral atoms. Second, the release energy from electron capture in a three-body recombination event (initial kinetic energy plus final binding energy) is transferred to the second involved electron and thus to the remaining quasi-free electron cloud. When removing the cold fraction of the quasi-free electrons, recombination effectively increases the electron temperature. In analogy to the release of latent heat during solidification of a liquid, the recombination induced energy release effectively slows down the expansion cooling of the electron gas. Thus recombination perpetuates the accelerating space charge field at the cluster surface for a longer time.

To substantiate these claims we performed semiclassical molecular dynamics simulations [34]. As a fully microscopic description of an $R=400 \mathrm{~nm}$ cluster is currently out of reach, $\mathrm{Xe}_{25000}(R \sim 7 \mathrm{~nm})$ was used as a smaller model system. The simulation results in Fig. 4 reproduce the main features of the measured ion spectra and confirm the key assumptions made to explain the physics. First, electron-ion recombination is highly efficient (see inset of Fig 4a) and strongly depletes the ion energy spectra in the low energy region [29], i.e. for ions from inner shells with high local electron density. This leads, as observed in the experiment, to the formation of isolated peaks for high charge states in the charge-state resolved spectra (Fig 4a). Note that recom- bination is not completed after the feasible simulation time of 4 ps. Further recombination in later stages is expected to result in isolated peaks for even lower charge states. Second, Figs. 4b) and 4c) contain a direct fingerprint of recombination-enhanced heating in the expanding nanoplasma. Whereas the number of localized electrons steadily increases due to recombination of onaverage hotter delocalized electrons, the temperature of the localized electrons keeps decreasing. This can only be explained by the preferential recombination of cold delocalized electrons, leaving behind an effectively hotter cloud of delocalized nanoplasma electrons that can release thermal energy to the ions via hydroexpansion. We like to stress that the simulated high-q ion spectra, for which the peak formation has taken place, have their maxima near $E / q \sim 100 \mathrm{eV}$ while the average thermal electron energy after excitation is below $30 \mathrm{eV}$, providing strong qualitative support for the claim of inhomogeneous and strongly enhanced energy redistribution to the surface ions.

In conclusion, we measured intensity, cluster size, and charge state selective ion spectra of large xenon clusters under intense femtosecond x-ray pulses and analyzed the resulting hydrodynamic cluster expansion process. We find a characteristic structure of the ion spectra with peak positions at finite energy, scaling linearly with charge state, and average ion energies well beyond the values expected for pure hydroexpansion. Our analysis of the energy spectra as well as MD simulations support that transient electron-ion recombination is responsible for both features. Most importantly, the measured high ion energies suggest that electron-ion recombination acts as a nanoplasma heating process by capturing the coldest part of the quasi-free electrons and releasing kinetic and binding energy to the remaining electron cloud. The observed nanoplasma expansion dynamics have direct implications for the physics of plasma expansion at surfaces $[54,55]$ and provide an important benchmark scenario for theory. The single shot capability of combined ion spectroscopy and x-ray imaging experiments will enable a deeper understanding of plasma expansion dynamics in weakly and strongly coupled plasmas. Including this novel acceleration mechanism into theoretical modeling will be important for experiments in a broad field from matter under extreme conditions to biophysics, aerosol science, and x-ray imaging of nanoparticles.

Excellent support from IOAP and DESY workshops is acknowledged. The experiments have been funded by BMBF (grants 05K10KT2/05K13KT2 from FSP FLASH) and DFG (grant BO 3169/2-2). T.F. acknowledges financial support by the DFG within SFB 652/3 and computer time provided by the North German Supercomputing Alliance (HLRN). 
*daniela.rupp@physik.tu-berlin.de

$\dagger$ thomas.fennel@uni-rostock.de

[1] W. Ackermann et al., Nat. Phot. 1, 336 (2007).

[2] P. Emma et al., Nat. Phot. 4, 641 (2010).

[3] E. Allaria, C. Callegari, D. Cocco, W. Fawley, M. Kiskinova, C. Masciovecchio, and F. Parmigiani, New J. Phys. 12, 075002 (2010).

[4] H. Chapman et al., Nat. Phys. 2 (2006).

[5] H. Chapman et al., Nature 470, 73 (2011).

[6] N. Loh et al., Nature, 513 (2012).

[7] D. Rupp et al., New J. Phys. 14, 055016 (2012).

[8] C. Bostedt et al., J. Phys. B 43, 194011 (2010).

[9] M. Seibert et al., Nature 470, 78 (2011).

[10] T. Ekeberg et al., Phys. Rev. Lett. 114, 098102 (2015).

[11] J. Küpper, S. Stern, L. Holmegaard, F. Filsinger, and A. Rouze et al., Phys. Rev. Lett. 112, 083002 (2014).

[12] S. Vinko et al., Nat. Comm. , 6397.

[13] E. M. Snyder, S. A. Buzza, and A. W. Castleman, Jr., Phys. Rev. Lett. 77, 3347 (1996).

[14] T. Ditmire, J. W. G. Tisch, E. Springate, M. Mason, N. Hay, R. A. Smith, J. Marangos, and M. H. R. Hutchinson, Nature 386, 54 (1997).

[15] M. Lezius et al., Phys. Rev. Lett. 80, 261 (1998).

[16] T. Ditmire, J. Zweiback, V. P. Yanovsky, T. E. Cowan, G. Hays, and K. B. Wharton, Nature 398, 489 (1999).

[17] V. Krainov and M. Smirnov, Physics Reports 370, 237 (2002).

[18] U. Saalmann, C. Siedschlag, and J. Rost, J. Phys. B 39, R39 (2006).

[19] T. Fennel, K.-H. Meiwes-Broer, J. Tiggesbäumker, P.-G. Reinhard, P. M. Dinh, and E. Suraud, Rev. Mod. Phys. 82, 1793 (2010).

[20] C. Bostedt, T. Gorkhover, D. Rupp, and T. Möller, in Synchrotron Light Sources and Free-Electron Lasers, edited by E. Jaeschke et al. (Springer, 2015).

[21] H. Wabnitz et al., Nature 420, 482 (2002).

[22] C. Bostedt et al., Nucl. Inst. Meth. 601, 108 (2009).

[23] C. Bostedt, H. Thomas, M. Hoener, T. Möller, U. Saalmann, I. Georgescu, C. Gnodtke, and J.-M. Rost, New J. Phys. 12, 083004 (2010).

[24] T. Laarmann, M. Rusek, H. Wabnitz, J. Schulz, A. R. B. de Castro, P. Gürtler, W. Laasch, and T. Möller, Phys. Rev. Lett. 95, 063402 (2005).

[25] C. Jungreuthmayer, L. Ramunno, J. Zanghellini, and T. Brabec, J. Phys. B 38, 3029 (2005).

[26] M. Hoener et al., J. Phys. B 41, 181001 (2008).

[27] H. Fukuzawa et al., Phys. Rev. A 79, 031201 (2009).

[28] B. Ziaja, H. Wabnitz, F. Wang, E. Weckert, and T. Möller, Phys. Rev. Lett. 102, 205002 (2009).
[29] M. Arbeiter and T. Fennel, New J. Phys. 13, 053022 (2011).

[30] S. Schorb, D. Rupp, M. L. Swiggers, R. N. Coffee, M. Messerschmidt, G. Williams, J. D. Bozek, S.-I. Wada, O. Kornilov, T. Möller, and C. Bostedt, Phys. Rev. Lett. 108, 233401 (2012).

[31] L. Schroedter et al., Phys. Rev. Lett. 112, 183401 (2014).

[32] Y. Ovcharenko et al., Phys. Rev. Lett. 112, 073401 (2014).

[33] M. Krikunova, M. Adolph, T. Gorkhover, D. Rupp, S. Schorb, C. Bostedt, S. Roling, B. Siemer, R. Mitzner, H. Zacharias, and T. Möller, J. Phys. B 45, 105101 (2012).

[34] M. Arbeiter, C. Peltz, and T. Fennel, Phys. Rev. A 89, 043428 (2014).

[35] T. Tachibana et al., Sci. Rep. 5, 10977 (2015).

[36] B. Schütte, M. Arbeiter, T. Fennel, M. J. J. Vrakking, and A. Rouzée, Phys. Rev. Lett. 112, 073003 (2014).

[37] B. Schütte, F. Campi, M. Arbeiter, T. Fennel, M. J. J. Vrakking, and A. Rouzée, Phys. Rev. Lett. 112, 253401 (2014).

[38] C. Peltz, C. Varin, T. Brabec, and T. Fennel, Phys. Rev. Lett. 113, 133401 (2014).

[39] T. Gorkhover et al., Nat. Phot. 10, 93 (2016).

[40] L. Flückiger et al., New J. Phys. 18, 043017 (2016).

[41] D. Rupp et al., J. Chem. Phys. 141, 044306 (2014).

[42] I. Barke et al., Nat. Comm. 6, 6187 (2015).

[43] L. Gomez et al., Science 6199, 906 (2014).

[44] C. Bostedt et al., Phys. Rev. Lett. 108, 093401 (2012).

[45] T. Gorkhover et al., Phys. Rev. Lett., 108, 245005 (2012).

[46] M. R. Islam, U. Saalmann, and J.-M. Rost, Phys. Rev. A 73, 041201 (2006).

[47] K. Ishikawa and T. Blenski, Phys. Rev. A 62, 063204 (2000).

[48] D. Hickstein et al., Phys. Rev. Lett. 112, 115004 (2014).

[49] A. A. Sorokin, S. V. Bobashev, T. Feigl, K. Tiedtke, H. Wabnitz, and M. Richter, Phys. Rev. Lett. 99, 213002 (2007).

[50] C. Bostedt et al., Phys. Rev. Lett. 100, 133401 (2008).

[51] See Supplemental Material [URL of supplemental material], which includes Refs. [56, 57].

[52] H. Thomas, C. Bostedt, M. Hoener, E. Eremina, H. Wabnitz, T. Laarmann, H. Plönjes, R. Treusch, A. de Castro, and T. Möller, J. Phys. B 42, 134018 (2009).

[53] M. Müller et al., J. Phys. B. 48, 174002 (2015).

[54] R. Snavely et al., Phys. Rev. Lett. 85, 2945 (2000).

[55] P. Mora, Phys. Rev. Lett. 90, 185002 (2003).

[56] SimIon version 8.0 (2013).

[57] I. Gilmore and M. Seah, International Journal of Mass Spectrometry 202, 217 (2000). 